\title{
Análisis de los tiempos de preparación para la reducción de desperdicios en el proceso de troquelado. Caso aplicado industria de calzado.
}

\section{Analysis of the preparation times for the reduction of waste in the die-cutting process. A case study in footwear industry}

Darwin Santiago Aldás

Miguel Patricio Barrionuevo Zurita

Universidad Técnica de Ambato, Ecuador

Narciza de Jesús Portalanza Molina,

Luis Patricio Tierra Pérez, Ingeniero

Escuela Superior Politécnica de Chimborazo, Ecuador

Autor para correspondencia: darwinsaldas@uta.edu.ec, nportalanza@espoch.edu.ec,

patricio.tierra@espoch.edu.ec, miguel_patricio91@hotmail.com

Fecha de recepción: 23 de abril de 2018 - Fecha de aceptación: 01 octubre de 2018

Resumen: En la presente investigación se analiza la reducción de desperdicios en los tiempos de preparación del proceso de troquelado en tres líneas de producción de industrias manufactureras de calzado de cuero. Se estudia los productos con mayor demanda a través de un análisis de segmentación de productos $\mathrm{ABC}$, una vez establecidos los mismos, se inicia con el levantamiento de información de todas las actividades que conforman el proceso de troquelado, realizando un estudio de tiempos, $\mathrm{y}$ luego un análisis acerca de las actividades en las que existen tiempos de preparación, para determinar los desperdicios dentro del proceso. En la investigación se utilizó la metodología SMED que busca reducir los tiempos de preparación transformando actividades internas del proceso en actividades externas. En el proceso de troquelado al poseer maquinas manuales se procede con la reducción de tiempos en las actividades de preparación de materiales y la estandarización de los procesos. Como resultados se obtuvieron reducción de desperdicios de tiempo en un 3\% en la línea de calzado casual, $6 \%$ en la línea de calzado deportivo de mujer y $10 \%$ en la línea de calzado de seguridad industrial en la producción diaria de cada uno de los procedimientos que comprenden el proceso de troquelado los mismos.

Palabras clave: SMED; troquelado; productividad; tiempos de preparación; desperdicios

Abstract: In the present investigation the reduction of waste in the preparation times is analyzed of the die-cutting process in three production lines of leather footwear manufacturing industries The products with the highest demand are studied through a segmentation analysis of ABC products, once established the same, begins with the gathering of information on all the activities that make up the die-cutting process, doing a study of times and then an analysis about the activities in which there are preparation times, to determine the waste within the process. In the investigation, the SMED methodology was used, which seeks to reduce preparation times by transforming internal activities of the process into external activities. In the process of die-cutting by owning manual machines we proceed with the reduction of time in the activities of preparation of materials and the standardization of the processes. As a result, a 3\% reduction in waste time was obtained in the casual footwear line, $6 \%$ in the line of sports shoes and $10 \%$ in the line of industrial safety in the daily production of each one of the procedures that comprise the die-cutting process.

Key Words: SMED; die-cutting; productivity; preparation times; waste 


\section{Introducción}

Hoy la industria de calzado ecuatoriano enfrenta problemas: alto costo de las materias primas, la falta de mano de obra calificada y la competencia desleal de quienes no cumplen las normas laborales, indican los productores. (El Universo, 2015). Los desafíos actuales deben orientar la conformación de nuevas industrias y la promoción de nuevos sectores con alta productividad, competitivos, sostenibles, sustentables y diversos, con visión territorial y de inclusión económica en los encadenamientos que generen. (Secretaría Nacional de Planificación y Desarrollo, 2013).

Con la finalidad de alcanzar los objetivos estratégicos la empresa, se ve en la necesidad de realizar estudios del trabajo para mejorar sus estándares, costos y ser más eficientes ante la creciente competencia y especificaciones de los clientes. (Reyes J. P., 2014).

La producción esbelta es una teoría fundamental para el mejoramiento de la productividad y la competitividad de las empresas de manufactura y servicios. La globalización de mercados exige a las organizaciones mantener estándares de clase mundial en sus procesos, entendiendo que toda ventaja competitiva es temporal y existe la necesidad de someterse a constantes procesos de adaptación por parte de todos los miembros de la red de valor (Morales, Rojas , \& Jimenez, 2015).

Se entiende por Lean Manufacturing ("producción ajustada") la persecución de una mejora del sistema de fabricación mediante la eliminación de desperdicios, entendiendo como desperdicio todas aquellas acciones que no aportan valor al producto y por las cuales el cliente no está dispuesto a pagar (Cerón Espinoza, Madrid Garcia, \& Gamboa Gómez , 2015). Es importante considerar que para que un sistema de manufactura sea optimo, la tasa de producción no debe variar en función del tiempo sino mantenerse constante, este es un claro indicador de que los recursos están llegando al sistema a tiempo y el aprovisionamiento del mismo es eficiente, donde se evita la generación de desperdicios. (Reyes , Aldás, \& Alvarez, 2017)

Dentro del análisis del tiempo productivo e improductivo se identifica a las horas de cambio como el principal motivo de improductividad, por esta razón se selecciona al sistema SMED como la herramienta de mejora a ser utilizada, cuyos principales beneficiarios son el aumento de la productividad, la reducción de costos en las ordenes de producción y el aumento de la flexibilidad de la línea de producción (Coello, 2007).

La técnica del SMED, aplicada a la preparación de equipos, máquinas o líneas de producción durante las actividades de cambio de modelo o producto, durante la ejecución de las actividades de mantenimiento preventivo, puede llevar a reducir hasta en un $60 \%$ los tiempos de parada programada de máquina (Sugai, Mcintosh, \& Novaski , 2007).

El sistema SMED (Single-Minute Exchange of Die) es un método probado que puede dar grandes resultados en una situación donde una máquina está involucrada en el proceso. Para la empresa, la adaptación de estas tres fases de mejora, conduce a un menor tiempo de preparación de máquinas, reduciéndose de modo que las líneas de producción tendrán una mayor disponibilidad, podrán trabajar con lotes más pequeños y asegurarán tiempos de entrega de producto menores (Alonzo Manzanedo, 2012). 
La técnica para reducir el tiempo de puesta en marcha utilizando SMED comienza con un análisis de producción e indicadores seguido de estudio de tiempo y luego el despliegue de todas las etapas de SMED. La primera actividad realizada es la observación, la segunda la evaluación y la implementación que condujo a la estandarización y a la generación de documentos de preparación. En este punto se realiza un entrenamiento que permite validar y evaluar la matriz de versatilidad, por último se evalúa la productividad de la célula de fabricación examinando el nuevo identificador de efectividad global para demostrar la eficacia del SMED en la reducción de los tiempos de puesta en marcha y ajuste fino de la maquinaria (Morales Mendez \& Silva Rodriguez, 2016).

La implementación de SMED permite entender la situación actual de las pérdidas de la planta ya que se logró identificar la mayor pérdida de la misma y las causas que la generaban. Asimismo me permite conocer al detalle el proceso de operación y puesta en marcha de una línea de tecnología de moldes tanto en sus aspectos operativos como de calidad y seguridad (Guerrero, 2008).

El objetivo principal es proponer la metodología para el análisis de configuración que se puede implementar principalmente en pequeñas y medianas empresas que no están convencidas de implementar el desarrollo de las configuraciones. (Stadnicka, 2015).

La industria de calzado nacional actualmente se encuentra en constante crecimiento, sin embargo, para alcanzar a los grandes productores mundiales y competir en los mercados internacionales es necesario elaborar estrategias de operaciones y sistemas productivos. Estas proporcionaran ventajas competitivas sobre los competidores extranjeros, con miras a tener implementado un modelo de gestión de procesos sostenible en cada industria (Reyes Vasquez, Aldas Salazar, Morales Perrazo , \& García Carrillo , 2016)

El problema principal radica en que las empresas no cuentan con un adecuado método de trabajo en especial en el proceso de troquelado que por ser donde inicia el proceso productivo genera en varias ocasiones retrasos que afectan a los demás procesos del sistema, así como también mayor generación de desperdicios de materia prima que representan pérdidas para las empresas, es por esto que se considera importante determinar y analizar los tiempos de preparación para una vez detectados, poder reducirlos y lograr elevar la productividad del sistema.

\section{Metodología}

Para el estudio se toman tres líneas de producción que son: Calzado casual (CSH), Calzado deportivo (DEM), Calzado de seguridad industrial(SI).La selección de los modelos o productos para el estudio se lo desarrolla en base al análisis $\mathrm{ABC}$, para el cual se recaba información del inventario de ventas del año 2017 de las empresas participantes; donde se establece el modelo del calzado, la cantidad de producto vendido y el costo unitario de cada producto. Los modelos seleccionados de cada línea de producción fueron elegidos en base al criterio 60 - 20 que se interpreta que el $60 \%$ de las ventas se dan por el $20 \%$ de productos 
vendidos. Luego de establecer el criterio se toma el producto estrella de cada una de las líneas de producción.

Posteriormente se realiza un estudio del trabajo en base a un enfoque cuali-cuantitativo para el proceso de montaje en la producción de calzado de cuero.

El estudio de métodos de trabajo se realiza de acuerdo al modelo seleccionado donde se aplican herramientas como el cursograma analítico o también conocido como diagrama de ensamble para determinar las principales operaciones e inspecciones que posea el proceso en análisis; de la misma manera se aplica el cursograma analítico donde se realiza un desglose de todas las actividades que conforman el troquelado de calzado. Para la medición del trabajo se escoge el estudio de tiempos con cronómetro vuelta a cero como la técnica para la medición del contenido de trabajo, con el objetivo de calcular el tiempo normal y el tiempo estándar que requiere un trabajador calificado para terminar una unidad en el proceso de troquelado, las etapas para realizar la medición del trabajo son:

Descomponer en elementos los procedimientos de troquelado

Toma de muestras de acuerdo a la tabla tomada de Time Study Manual de los Eric Works de General Electric Company que en nuestro caso señala 10 muestras si el tiempo estándar no supera los 5 minutos.

Cronometraje de la operación, para lo cual se opta por el cronometraje vuelta a acero.

- Valoración del trabajador de acuerdo a los criterios de la tabla Británica.

- Cálculo del tiempo normal o básico. Ver ecuación 2

$$
T B=\frac{\text { Tiempo observado } * \text { Valor del ritmo observado }}{\text { Valor del ritmo tipo }}
$$

- Cálculo de suplementos por descanso; fijos y variables

- Cálculo del tiempo estándar mediante el uso de la ecuación 3

Tiempo estándar $=\{$ Tiempo normal $\} *(1+$ suplementos $)$

En el presente estudio de analiza solamente la sección de corte ya que esta al ser la primera en todo el proceso, puede generar retrasos en las otras actividades. Se toma en cuenta dos actividades fundamentales del proceso que son corte de cuero que es la materia prima misma y corte de complementos que son todos los insumos adicionales como forros, celfil, telas, esponjas.

\section{Aplicación de la Metodología SMED}

La metodología SMED la integra 3 fases, donde la primera identifica las operaciones internas y externas en el proceso de preparación de maquinaria. La segunda fase plantea que se debe convertir las actividades internas en externas y la tercera es la mejora tanto en las actividades internas como externa. En el proceso de troquelado no requiere la calibración de la máquina para cada cambio de modelo, con lo cual no se realizan las dos primeras fases que plantea la metodología SMED ya que la mayoría de preparaciones es de manera manual, es por ello que se aplica la tercera fase que es la mejora y estandarización de actividades. 
En la Tabla 3 se presenta el desglose de actividades de preparación de materiales para el corte de cuero y complementos de las cuales se seleccionaran tareas que se pueden eliminar o delegar la tarea a un operario auxiliar. Las tareas tachadas son las que se reducirán u otorgarán a otro operario para su desarrollo.

En el presente estudio se descompone el proceso de troquelado en dos operaciones principales que son corte de cuero y corte de complementos, las actividades de la operación de corte de cuero con sus respectivos tiempos del modelo calzado casual CSH se muestra en la tabla 1, en la misma que se indica los tiempos de preparación, siendo éstas preparación de consumos, preparación de cantidad de bandas necesarias para lote requerido y preparación de moldes para realizar cortes

Tabla 1: Tiempos de operación de corte de cuero en minutos

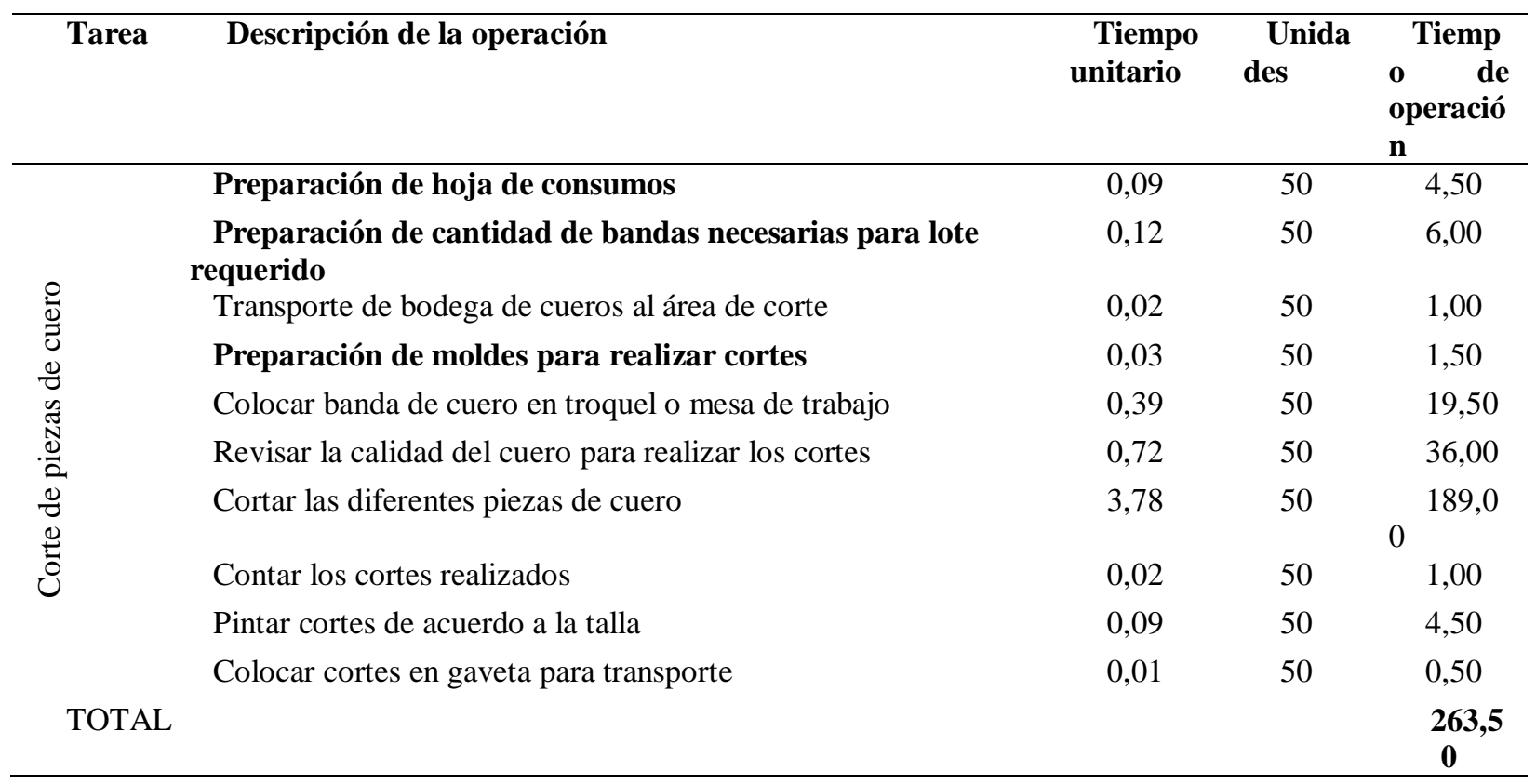

Para reducir cada uno de los tiempos de preparación se descompone las mismas en sub actividades descritas en la tabla 2, tabla 3 y tabla 4 .

En el proceso de corte de cueros se reducen los tiempos de preparación de hoja de consumo, preparación de bandas de cuero y la preparación de los moldes para los cortes, de cada una de estas actividades se realiza un desglose de las tareas que comprenden dichas actividades para analizar cuáles pueden ser reducidas.

La preparación de la hoja de consumo comprende de 5 actividades de las cuales se redujo el tiempo de revisión de existencia de material solicitado manteniendo el inventario de materia prima actualizado, también se redujo el tiempo que demora llenar la hoja de consumos mediante la impresión directa de los datos de consumo desde el sistema. En la preparación de las bandas de cuero se cambiaría al encargado de bodega para que el operario una vez generada la orden de producción tenga listo el material necesario para el trabajador reduciendo totalmente el tiempo 
de preparación. Para la preparación de los moldes para el troquelado de las piezas de cuero deben ser correctamente etiquetados o colocados en orden de acuerdo al modelo y talla, lo que agilitará el proceso de búsqueda de los mismos agilitando el trabajo del operario.

Tabla 2: Sub actividades de preparación de consumos en minutos

\begin{tabular}{|c|c|c|c|c|}
\hline Tarea & Descripción de la operación & $\begin{array}{l}\quad \text { Tiempo } \\
\text { unitario } \\
\text { (min) }\end{array}$ & Unidades & $\begin{array}{l}\text { Tiempo } \\
\text { de } \\
\text { operación } \\
(\text { min) }\end{array}$ \\
\hline \& & Recepción de la orden de producción & 0,005 & 50 & 0,24 \\
\hline \multirow{5}{*}{ 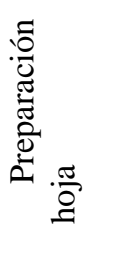 } & Ingreso de la orden de producción al sistema & 0,030 & 1 & 1,48 \\
\hline & Consulta del modelo solicitado & 0,013 & 1 & 0,67 \\
\hline & $\underline{\text { Revisión de existencia de material solicitado }}$ & $\underline{0,012}$ & $\underline{1}$ & 0,59 \\
\hline & $\underline{\text { Llenar la hoja de consumos con los datos del Sistema }}$ & $\underline{0,030}$ & $\underline{1}$ & 1,52 \\
\hline & & Total & & 2,39 min \\
\hline
\end{tabular}

Tabla 3. Desglose de actividades preparación bandas de cuero

\begin{tabular}{|c|c|c|c|c|}
\hline a & Descripción de la operación & $\begin{array}{l}\quad \text { Tiemp } \\
\text { o } \\
\text { unitario } \\
\text { (min.) }\end{array}$ & $\begin{array}{l}\text { Unidad } \\
\text { es }\end{array}$ & $\begin{array}{l}\text { Tiemp } \\
\text { o de } \\
\text { operació } \\
\text { n (min) }\end{array}$ \\
\hline 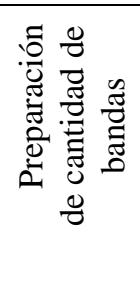 & $\begin{array}{l}\text { Abrir empaque de bandas de cuero } \\
\underline{\text { Sumar decímetros de cada banda hasta completar consumo }} \\
\text { necesario } \\
\underline{\text { Empacar bandas sobrantes para almacenaje }} \\
\text { TOTAL }\end{array}$ & $\begin{array}{l}\frac{0,024}{2} \\
\underline{0,072} \\
\underline{0,023} \\
\underline{8}\end{array}$ & $\begin{array}{l}\underline{50} \\
\underline{50}\end{array}$ & $\begin{array}{c}3,6 \\
1,19 \\
0 \text { min }\end{array}$ \\
\hline
\end{tabular}

Tabla 4. Desglose de actividades preparación moldes

\begin{tabular}{|c|c|c|c|c|}
\hline Tarea & Descripción de la operación & $\begin{array}{c}\text { Tiempo } \\
\text { unitario }\end{array}$ & Unidades & $\begin{array}{l}\text { Tiempo de } \\
\text { operación }\end{array}$ \\
\hline \multirow{4}{*}{ 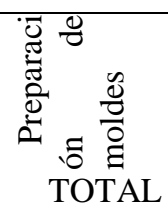 } & Recepción de la hoja de consumo y bandas de cuero & 0,0054 & 50 & 0,27 \\
\hline & Búsqueda de moldes para realizar cotes & 0,0196 & 50 & 0,98 \\
\hline & Verificar moldes de acuerdo a las tallas solicitadas & 0,005 & 50 & 0,25 \\
\hline & & & & $1,25 \mathrm{~min}$ \\
\hline
\end{tabular}


Antes de aplicar la metodología SMED, el tiempo que tardaban en preparación para la actividad de corte de cuero era de $12 \mathrm{~min}$, y en el propuesto es de 3, $64 \mathrm{~min}$, logrando una reducción de $\mathbf{8 , 3 6} \mathbf{m i n}$ por lote.

De la igual forma se divide la operación de corte de complementos en tres sub actividades que son : preparación de complementos requeridos y preparación de moldes para corte de complementos Tabla 5, las mismas que están descompuestas en sub actividades mostradas en la tabla 6 y 7.

Tabla 5. Tiempo estándar de actividad (min)

\begin{tabular}{|c|c|c|c|c|}
\hline Tarea & Descripción de la operación & $\begin{array}{l}\text { Tiempo } \\
\text { unitario }\end{array}$ & Unidades & $\begin{array}{l}\text { Tiempo de } \\
\text { operación }\end{array}$ \\
\hline \multirow{10}{*}{ 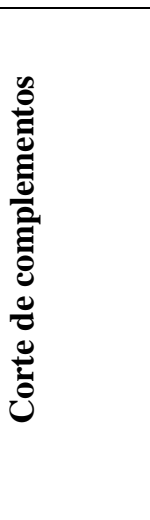 } & Preparación de complementos requeridos & 0,09 & 50 & 4,50 \\
\hline & Transporte de materiales al área de corte & 0,17 & 50 & 8,50 \\
\hline & Espera de operario & 0,02 & 50 & 1,00 \\
\hline & Preparación de moldes para corte de complementos & 0,04 & 50 & 2,00 \\
\hline & Corte de forros y telas & 0,60 & 50 & 30,00 \\
\hline & Corte de Esponjas & 0,22 & 50 & 11,00 \\
\hline & Corte de Celfil (Troquel) & 0,37 & 50 & 18,50 \\
\hline & Contar cortes Requeridos & 0,08 & 50 & 4,00 \\
\hline & Hacer lotes de los complementos & 0,07 & 50 & 3,50 \\
\hline & Colocar lotes de complementos en gaveta de transporte & 0,02 & 50 & 1,00 \\
\hline TOTAI & & & \multicolumn{2}{|c|}{84,00} \\
\hline
\end{tabular}

En el caso de los complementos se ha eliminado las actividades que se deben realizar por los encargados de bodega como la preparación de las cantidades necesarias de materiales para que el operario se dedique netamente a su trabajo que es el corte.

Tabla 6. Desglose de actividades preparación de complementos

\begin{tabular}{|c|c|c|c|c|}
\hline Tarea & Descripción de la operación & Tiempo unitario & Unidades & Tiempo de operación \\
\hline \multirow{5}{*}{ 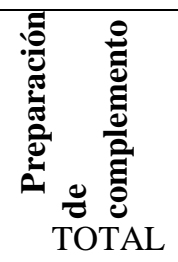 } & $\underline{\text { Recepción de hoja de consumos }}$ & 0,0036 & 1 & 0,18 \\
\hline & $\underline{\text { Medición y pliegue de Forro }}$ & 0,0244 & 1 & 1,22 \\
\hline & $\underline{\text { Medición y pliegue de Tela }}$ & 0,0262 & 1 & 1,31 \\
\hline & Medición y Pliegue de esponja & 0,0358 & 1 & 1,79 \\
\hline & & & & 0 \\
\hline
\end{tabular}


Tabla7. Desglose de actividades preparación de moldes

\begin{tabular}{|c|c|c|c|c|}
\hline Tarea & Descripción de la operación & $\begin{array}{l}\text { Tiempo } \\
\text { unitario }\end{array}$ & Unidades & $\begin{array}{l}\text { Tiempo de } \\
\text { operación }\end{array}$ \\
\hline \multirow{2}{*}{ 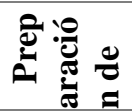 } & Búsqueda de moldes para realizar cotes & 0,029 & 50 & 1,46 \\
\hline & Verificar moldes de acuerdo a las tallas solicitadas & $\theta, 011$ & 50 & $\theta, 54$ \\
\hline
\end{tabular}

Antes de aplicar la metodología SMED, el tiempo que tardaban en preparación para la actividad de corte de complementos de cuero era de 6,5 min, y en el propuesto es de 1, 46 min, logrando una reducción de 5,04 min por lote.

En los dos modelos siguientes, calzado deportivo de mujer DEM y calzado de seguridad industrial SI se aplica la misma metodología, quedando en resumen los siguientes tiempos, que se muestran en las Tablas 8 y 9.

Tabla 8. Resumen reducción de tiempos de preparación calzado DEM

\begin{tabular}{lccc}
\hline \multicolumn{2}{c}{ Calzado Deportivo de mujer (DEM) (Tiempos en minutos) } \\
Actividad de preparación & \multicolumn{2}{c}{ Corte de cuero } & \\
\cline { 2 - 3 } & Tiempo & Tiempo & Tiempo \\
& actual & SMED & reducido \\
Preparación de hoja de consumos & 4,29 & 2,87 & 1,42 \\
Preparación de cantidad de bandas necesarias para & 6,80 & 0 & 6,80 \\
lote requerido & 2,19 & 1,71 & 0,48 \\
Preparación de cortes para realizar cortes & 13,28 & 4,58 & 8,7 \\
Total & Corte de Complementos & \\
Actividad de preparación & & & \\
& Tiempo & Tiempo & Tiempo \\
& actual & SMED & reducido \\
Preparación de complementos requeridos & 5,66 & 0 & 5,66 \\
Preparación de moldes para corte de complementos & 1,36 & 0,94 & 0,42 \\
Total & 7,02 & 0,94 & 6,08 \\
\hline
\end{tabular}

Tabla 9. Resumen reducción de tiempos de preparación calzado SI

Calzado de Seguridad (SI) (Tiempos en minutos)

Actividad de preparación
Corte de cuero

\begin{tabular}{|c|c|c|c|}
\hline & $\begin{array}{l}\text { Tiempo } \\
\text { actual }\end{array}$ & $\begin{array}{l}\text { Tiempo } \\
\text { SMED }\end{array}$ & $\begin{array}{l}\text { Tiempo } \\
\text { reducido }\end{array}$ \\
\hline $\begin{array}{l}\text { Preparación de cantidad de bandas necesarias para lote } \\
\text { requerido }\end{array}$ & 12,36 & 0 & 12,36 \\
\hline Preparación de cortes para realizar cortes & 4,40 & 3,46 & 0,94 \\
\hline Total & 16,76 & 3,46 & 13,30 \\
\hline Actividad de preparación & \multicolumn{3}{|c|}{ Corte de Complementos } \\
\hline
\end{tabular}


Preparación de complementos requeridos

Preparación de moldes para corte de complementos

Total

Tiempo
actual
13,24
5,57
18,81

Tiempo
SMED

0

4,83

4,83
Tiempo reducido

13,24

0,74

13,98

Una vez aplicada la metodología SMED en el proceso de troquelado en las tres líneas de calzado seleccionadas, se procede con la comparación de los tiempos obtenidos en las operaciones de preparación como se indica en la Tabla 10.

Tabla10. Resultados obtenidos en los procedimientos de Troquelado

\begin{tabular}{lllccl}
\hline Modelo & Actividad & $\begin{array}{c}\text { Tiempo } \\
\text { inicial (min) }\end{array}$ & $\begin{array}{c}\text { Tiempo de } \\
\text { SMED(min) }\end{array}$ & $\begin{array}{c}\text { Tiempo } \\
\text { mejorado } \\
\text { (min) }\end{array}$ & $\begin{array}{c}\text { Porcentaje de } \\
\text { reducción }\end{array}$ \\
\hline CSH & Corte de Cuero & 263,50 & 255,14 & 8,36 & \\
& Corte de Complementos & 84,00 & 78,96 & 5,04 & $\mathbf{6 \%}$ \\
DEM & Corte de cuero & 134,02 & 125,32 & 8,70 & $\mathbf{6 \%}$ \\
& Corte de complementos & 100,17 & 94,09 & 6,08 & $\mathbf{6 \%}$ \\
SI & Corte de cueros & 131,76 & 118,46 & 13,30 & $\mathbf{1 0 \%}$ \\
& Corte de Complementos & 88,40 & 74,42 & 13,98 & $\mathbf{1 6 \%}$ \\
\end{tabular}
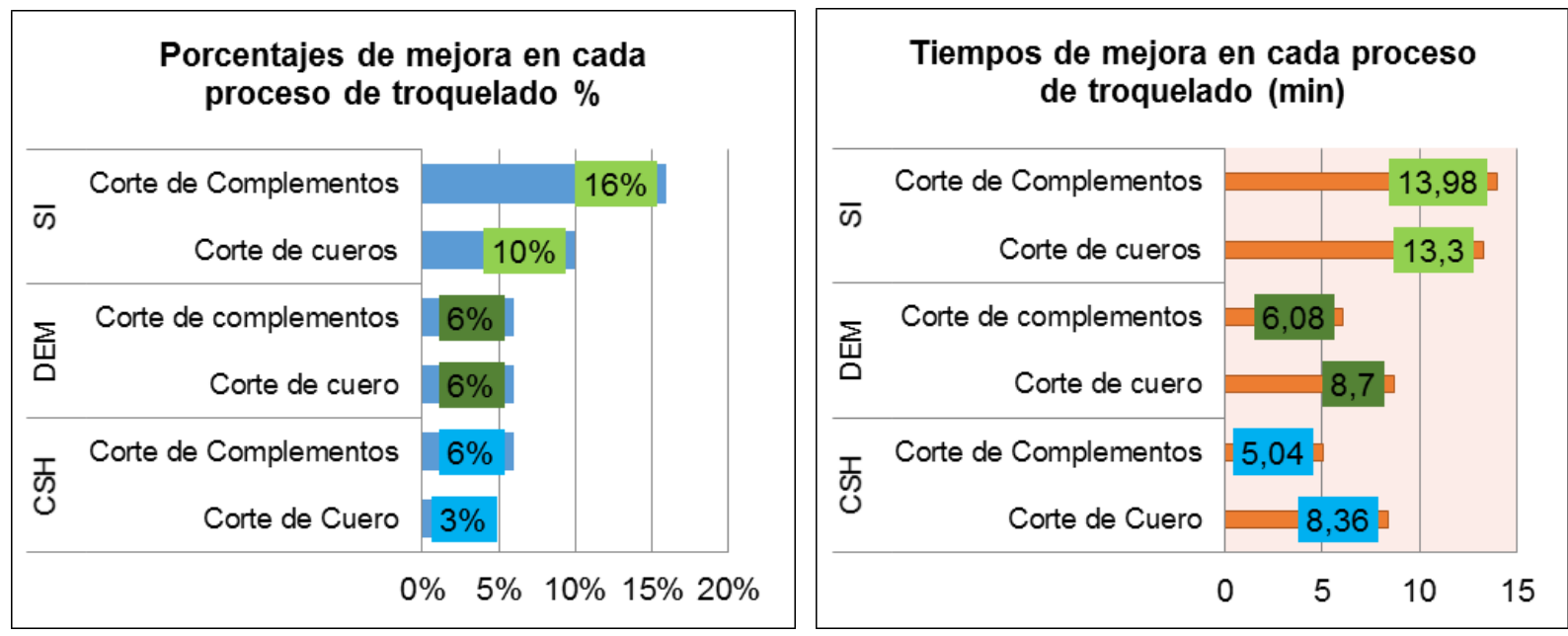

Figura1. Cuadro comparativo de resultados 


\section{Conclusiones}

Entre los principales desperdicios encontrados en la actividad de corte de cueros y corte de complementos tenemos los tiempos de preparación de materiales, en los cuales se encuentran la preparación de las bandas de cuero, los moldes para realizar cortes, la generación de órdenes de producción, en el modelo de calzado casual se tiene un total de tiempo de preparación de 18,5 minutos por lote de zapatos, en la línea de calzado deportivo tenemos un tiempo de preparación de 20,3 minutos por lote de zapatos y en el modelo de calzado de seguridad industrial tenemos un tiempo de preparación de 35,57 minutos por lote, los mismos que deben ser reducidos mediante la aplicación de la metodología SMED con la aplicación de la estandarización de las tareas.

Con la aplicación de la metodología SMED y la estandarización de las tareas se redujo el tiempo de producción de calzado para la operación de corte de cuero en un lote de 50 pares para el modelo calzado casual en 5,04 min correspondiente al 6\%, en el calzado deportivo de mujer de redujo el tiempo en 8,7 min que equivale al $6 \%$ de todo el proceso y en la línea de calzado de seguridad industrial en 13, 3 min equivalente al $10 \%$. Como son procesos estandarizados, estos porcentajes son aplicables a otros números e lotes de producción.

Así también Con la aplicación de la metodología SMED se redujo el tiempo de producción de calzado para la operación de corte de complementos en un lote de 50 pares para el modelo calzado casual en 8,36 min correspondiente al 3\%, en el calzado deportivo de mujer de redujo el tiempo en 6,08 min que equivale al $6 \%$ de todo el proceso y en la línea de calzado de seguridad industrial en 13, 98 min equivalente al $16 \%$. Como son procesos estandarizados, estos porcentajes son aplicables a otros números e lotes de producción.

Este estudio es aplicable a diversas empresas de calzado, ya que en el estudio se tomaron las líneas de producción más demandadas por el mercado y las actividades analizadas son estándares para la mayoría de procesos de producción de calzado en el área de corte o troquelado. 


\section{Bibliografía}

Alonzo Manzanedo, H. H. (2012). Optimización de operaciones mediante la técnica SMED en una empresa de envases metálicos. Ingeniería Industrial, 1543-1541.

Cerón Espinoza, J., Madrid Garcia, J., \& Gamboa Gómez , A. (2015). Desarrollo y casos de aplicación de Lean Manufacturing. Magazín Empresarial, 1(28).

Chacon , A., Fajardo , H., Fernandez , A., \& Gonzales , J. (29 de July de 2015). Gestiopolis. Obtenido de https://www.gestiopolis.com/smed-single-minute-exchange-die-conceptosfundamentales/

Coello, J. I. (2007). Diseño del Sistema SMED en el proceso de troquelado en una empresa de Artes gráficas. Guayaquil: Escuela Superior Politecnica Del Litoral. Recuperado el 10 de Marzo de 2016, de http://www.dspace.espol.edu.ec/xmlui/bitstream/handle/123456789/4300/6820.pdf?seque nce $=1 \&$ isAllowed $=\mathrm{y}$

El Universo. (19 de Julio de 2015). Innovación y diseño son aún un desafío para el zapato ecuatoriano. El universo.

Guerrero, G. A. (2008). Aplicación de la metodología SMED para la reducción de los tiempos de cambio de formato en una línea de producción de helados. Guayaquil: Escuela Superior Politecnica Del Litoral.

Morales Mendez, J., \& Silva Rodriguez, R. (2016). Set-up reduction in an interconecction axle manufacturing cell using SMED. 84(9), 1907- 1916.

Morales, A., Rojas , J., \& Jimenez, M. (2015). Modelo de un sistema de producción esbelto con redes de Petri para apoyar la toma de decisiones. Ingeniare. Revista Chilena de Ingeniería, 23(2).

Moyano Jose, M. P. (2012). El papel de las tecnologías de la información y las comunicaciones (TIC) en la búsqueda de la eficiencia, un análisis desde Lean Production y la integración electrónica de la cadena de suministro. Cuadernos de economía y dirección de empresas CEDE, 15(03).

Reyes , J., Aldás, D., \& Alvarez, K. (2017). The Factory Physics for the Scheduling: Aplication to Footwear Industry. SIMULTECH 2017, 248-254.

Reyes Vasquez, J., Aldas Salazar, D., Morales Perrazo , L., \& García Carrillo , M. (2016). Evaluación de la capacidad para montaje en la industria manufacturera de calzado. Ingeniería Industrial, 37(1). 
Reyes, J. P. (2014). Estudio de métodos y tiempos para montaje de Calzado. En Estudio del Trabajo. Aplicaciones en la Industria Ecuatoriana (págs. 90-92). Ambato, Tungurahua, Ecuador.

Secretaría Nacional de Planificación y Desarrollo. (2013). Todo el mundo mejor. Buen Vivir Plan Nacional, 1, 600 .

Stadnicka, D. (2015). Setup Analysis: Combining SMED with Other Tools. Management and Production Engineering Review, 6(1), 36-50.

Sugai, M., Mcintosh, R., \& Novaski , O. (2007). Metodología Shigeo Shingo ( SMED ) : análisis crítico y estudio de casos. Gestión y producción, 14(02). 